\title{
Oxidative Addition of Diethylchalcogenanes to Lapperts Germylene and Stannylene
}

\author{
Georg Bendt, Silvia Lapsien, Phillip Steiniger, Dieter Bläser, Christoph Wölper and Stephan \\ Schulz*
}

Keywords: Germylene, Stannylene, Dichalcogenanes, Main Group Chemistry

Oxidative addition of diethyldichalcogenanes $\mathrm{Et}_{2} \mathrm{E}_{2}(\mathrm{E}=\mathrm{S}, \mathrm{Se}, \mathrm{Te})$ to bis[bis(trimethylsilyl]amido]germylene and -stannylene $\mathrm{M}\left[\mathrm{N}\left(\mathrm{SiMe}_{3}\right)_{2}\right]_{2} \quad(\mathrm{M}=\mathrm{Ge}, \mathrm{Sn})$ proceed with formation of the corresponding bis(ethylchalcogenato)germanes

\author{
$\left(\mathrm{Me} 3 \mathrm{Si}_{2} \mathrm{~N}\right)_{2} \mathrm{Ge}(\mathrm{EEt})_{2}(\mathrm{E}=\mathrm{S} \mathrm{1}, \mathrm{Se} 2$, Te 3) and -stannanes \\ $\left.\left(\mathrm{Me}_{3} \mathrm{Si}\right)_{2} \mathrm{~N}\right)_{2} \mathrm{Sn}(\mathrm{EEt})_{2}(\mathrm{E}=\mathrm{S}$ 4, Se 5, Te 6). The solid structures \\ of $\mathbf{1}$ - $\mathbf{6}$ were determined by single crystal X-ray diffraction.
}

* Prof. Dr. Stephan Schulz Fax: + 49 0201-1834635, Phone: + 4902011834635 stephan.schulz@uni-due.de

Faculty of Chemistry, University of Duisburg-Essen, 45117 Essen, Germany

E-mail: Homepage: http://www.uni-due.de/ak_schulz/

$\square \quad$ Supporting information for this article is available on the WWW under http://dx.doi.org/10.1002/zaac.201000xxx or from the author.

\section{Introduction}

In recent years, the heavier analogues of carbenes $\mathrm{R}_{2} \mathrm{C}$, i.e., silylenes $\mathrm{R}_{2} \mathrm{Si}$, germylenes $\mathrm{R}_{2} \mathrm{Ge}$, stannylenes $\mathrm{R}_{2} \mathrm{Sn}$, and plumbylenes $\mathrm{R}_{2} \mathrm{~Pb}$ have received increasing interest. ${ }^{[1]}$ These divalent species, in which the group 14 element adopts the formal oxidation state + II and whose stability increases with increasing number of the group 14 element, typically have singlet ground states due to the somewhat larger energy gap between the $n \mathrm{~s}$ and $n \mathrm{p}$ orbitals of the heavier group 14 elements $(\mathrm{n}=3,4,5,6)$ compared to carbon $(n=2)$. As a consequence, two electrons are located in a low lying s-orbital (electron lone-pair) while the higherlying p-orbital is empty. ${ }^{[2]}$ As a consequence of this specific electronic situation, the heavier analogues of carbenes can simultaneously react as Lewis acid and as Lewis base. In particular silylenes and germylenes have evolved from exotic reaction intermediates to important reagents in organic chemistry and they were shown to activate a large variety of small molecules. ${ }^{[3]}$

Due to our general interest in the synthesis, structure and reactivity of complexes containing a direct group 14/16 and group 15/16 bond, which are also promising single source precursors for the generation of the corresponding binary materials, ${ }^{[4]}$ we recently started our studies on the synthesis of $\mathrm{Ge} / \mathrm{Sn}$-chalcogenide complexes. ${ }^{[5]} \mathrm{SnSe}$ has only recently received particular interest due to its very promising thermoelectric properties. ${ }^{[6]}$ Reactions of germylenes $\mathrm{R}_{2} \mathrm{Ge}$ with chalcogenes typically occur with formation of complexes containing chalcogen-bridges, ${ }^{[7]}$ terminal $\mathrm{Ge}=\mathrm{E}$ bonds ${ }^{[8]}$ or polychalcogenides. ${ }^{[9]}$ Comparable findings were reported for analogous reactions of stannylenes $\mathrm{R}_{2} \mathrm{Sn}^{\left[{ }^{[10]} \mathrm{In}\right.}$ addition, reactions of $R_{2} \mathrm{M}$ with dichalcogenanes $\mathrm{R}_{2} \mathrm{E}_{2}$ are known to proceed with insertion into the E-E bond of the formation of complexes of the type $\mathrm{R}_{2} \mathrm{M}\left(\mathrm{ER}^{\prime}\right)_{2}(\mathrm{M}=\mathrm{Ge}, \mathrm{Sn}$ $\mathrm{E}=\mathrm{S}, \mathrm{Se}, \mathrm{Te}){ }^{[10 \mathrm{a}, 11]}$ Moreover, insertion reactions of monovalent tin (RSnSnR), antimony (RSb), bismuth (RBi) and zinc complexes $\left(R_{2} Z_{2}\right)$ in the E-E bond of $R_{2} E_{2}$ were recently reported. ${ }^{[12]}$

We recently reported on reactions of the cyclic bis(amino)germylene $\quad\left[\mathrm{Me}_{2} \mathrm{Si}(\mathrm{N} t-\mathrm{Bu})_{2}\right] \mathrm{Ge} \quad$ with dialkyldichalcogenanes $\mathrm{R}_{2} \mathrm{E}_{2}(\mathrm{E}=\mathrm{S}, \mathrm{Se}, \mathrm{Te} ; \mathrm{R}=\mathrm{Me}, \mathrm{Et})$, which proceeded with formation of the expected germanium dichalcogenides $\mathrm{Me}_{2} \mathrm{Si}(\mathrm{N} t-\mathrm{Bu})_{2} \mathrm{Ge}(\mathrm{ER})_{2}$ as well as of the unexpected digermanes $\left[\mathrm{Me}_{2} \mathrm{Si}(\mathrm{N} t-\mathrm{Bu})_{2} \mathrm{Ge}(\mathrm{ER})\right]_{2}$, respectively. ${ }^{[13]}$ In order to further investigate this unexpected reaction behavior, we expanded our studies on analogous reactions of the acyclic bis[bis(trimethylsilyl]amido]germylene $\left.\left(\mathrm{Me}_{3} \mathrm{Si}\right)_{2} \mathrm{~N}\right)_{2} \mathrm{Ge}$ as well as on the corresponding bis[bis(trimethylsilyl]amido]stannylene $\left.\left(\mathrm{Me}_{3} \mathrm{Si}\right)_{2} \mathrm{~N}\right)_{2} \mathrm{Sn} .{ }^{[14]}$

\section{Results and Discussion}

The reactions of $\left[\left(\mathrm{Me}_{3} \mathrm{Si}\right)_{2} \mathrm{~N}\right] \mathrm{Ge}$ with $\mathrm{Et}_{2} \mathrm{E}_{2}(\mathrm{E}=\mathrm{S}, \mathrm{Se}, \mathrm{Te})$ in hexane at ambient temperature led to the quantitative formation of $\left[\left(\mathrm{Me}_{3} \mathrm{Si}\right)_{2} \mathrm{~N}\right] \mathrm{Ge}(\mathrm{EEt})_{2}(\mathrm{E}=\mathrm{S} \mathrm{1}$, Se 2, Te 3).
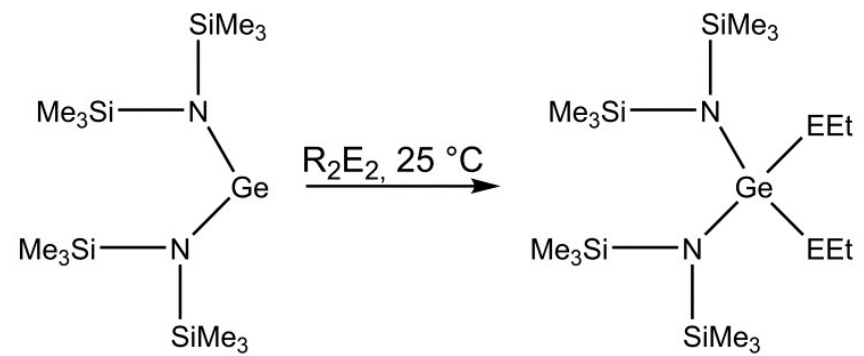

$\mathrm{E}=\mathrm{S} \mathbf{1}, \mathrm{Se} 2 ; \mathrm{Te} \mathbf{3}$

Scheme 1. Synthesis of $\mathbf{1}-\mathbf{3}$

The progress of the reactions could be easily followed since a color change from yellow to colorless (1 and 2) as well as red to yellow (3), respectively, occurred within a 
minute. ${ }^{[15]}{ }^{1} \mathrm{H}$ and ${ }^{13} \mathrm{C}$ NMR spectra of the resulting products only showed the expected resonances due to the Et and $\mathrm{N}\left(\mathrm{SiMe}_{3}\right)_{2}$ groups in the expected 1:1 molar ratios, indicating the insertion of the germylene into the E-E bond with subsequent formation of the corresponding germanes. This is in remarkable contrast to the reactions between $\mathrm{Et}_{2} \mathrm{E}_{2}$ and the cyclic germylene $\left[\mathrm{Me}_{2} \mathrm{Si}(\mathrm{N} t-\mathrm{Bu})_{2}\right] \mathrm{Ge}$, in which the digermanes $\left[\mathrm{Me}_{2} \mathrm{Si}(\mathrm{N} t-\mathrm{Bu})_{2} \mathrm{GeEEt}\right]_{2}$, containing a direct $\mathrm{Ge}-$ $\mathrm{Ge}$ bond and the $\mathrm{Ge}$ atoms adopting the formal oxidation state +III, were unexpectedly formed in addition to the expected germanes $\left[\mathrm{Me}_{2} \mathrm{Si}(\mathrm{N} t-\mathrm{Bu})_{2}\right] \mathrm{Ge}(\mathrm{EEt})_{2}$, in which the Ge atoms adopt the preferred formal oxidation state $+\mathrm{IV} .^{[13]}$ In addition, the ${ }^{77}$ Se NMR and ${ }^{127}$ Te NMR spectra of $\mathbf{2}$ and $\mathbf{3}$ also showed only a single resonance (table 1 ).

Table 1. NMR resonances of $\mathbf{1}$ - $\mathbf{6}$ [ppm]. ${ }^{[\mathrm{a}]}$

\begin{tabular}{lllllll}
\hline & $\mathbf{1}$ & $\mathbf{2}$ & $\mathbf{3}$ & $\mathbf{4}$ & $\mathbf{5}$ & $\mathbf{6}$ \\
\hline${ }^{1} \mathrm{H}$ & $2.77(\mathrm{q})$ & $2.77(\mathrm{q})$ & $2.71(\mathrm{q})$ & $2.82(\mathrm{q})$ & $2.83(\mathrm{q})$ & $2.76(\mathrm{q})$ \\
& $1.20(\mathrm{t})$ & $1.29(\mathrm{t})$ & $1.45(\mathrm{t})$ & $1.23(\mathrm{t})$ & $1.31(\mathrm{t})$ & $1.46(\mathrm{t})$ \\
& $0.47(\mathrm{~s})$ & $0.49(\mathrm{~s})$ & $0.50(\mathrm{~s})$ & $0.42(\mathrm{~s})$ & $0.48(\mathrm{~s})$ & $0.45(\mathrm{~s})$ \\
${ }^{13} \mathrm{C}$ & 6.6 & 6.7 & 4.1 & 6.2 & 6.5 & -0.6 \\
& 17.2 & 17.0 & 7.0 & 18.7 & 17.3 & 6.8 \\
& 24.7 & 19.9 & 17.7 & 23.4 & 18.4 & 19.0 \\
${ }^{77} \mathrm{Se}$ & - & 124.7 & - & - & 39.7 & - \\
${ }^{119} \mathrm{Sn}$ & - & - & - & 699.2 & 699.2 & 699.2 \\
${ }^{127} \mathrm{Te}$ & - & - & 162.3 & - & - & -15.5 \\
\hline
\end{tabular}

[a] NMR spectra were recorded in $\mathrm{C}_{6} \mathrm{D}_{6}$.

Single crystals of $\mathbf{1}$ - $\mathbf{3}$ were obtained upon storage of solutions of $\mathbf{1}-\mathbf{3}$ in hexane at $-30{ }^{\circ} \mathrm{C}$ for $12 \mathrm{~h} . \mathbf{1}$ and $\mathbf{2}$ are isostructural and crystallize in the triclinic space group $P-1$ with two independent molecules in the asymmetric unit. 3 crystallizes in the monoclinic space group $C 2 / c$ with the molecule placed on a two-fold axis.
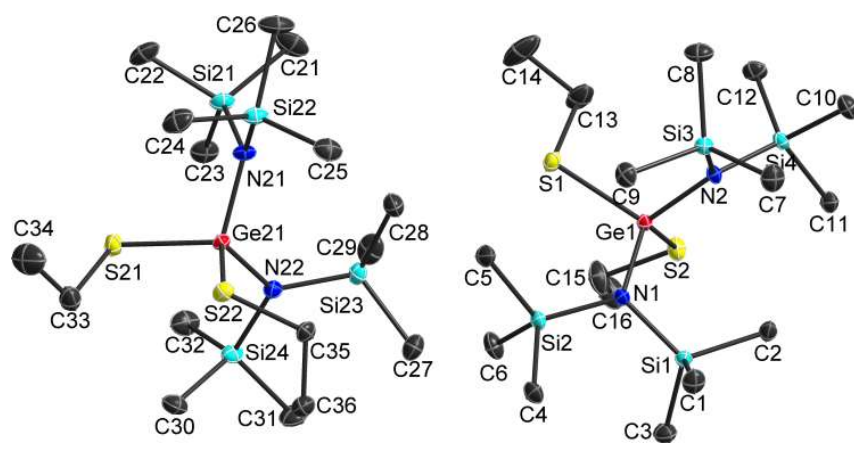

Figure 1. Solid state structure of $\mathbf{1}$. Non-H-atoms shown as thermal ellipsoids at $50 \%$ probability levels; $\mathrm{H}$ atoms and second orientation of disordered parts omitted for clarity.

The average Ge-E bond lengths (av. 2.2335(5) $\AA$ 1; $2.3707(4) \AA 2$; 2.5803(2) $\AA$ 3) agree perfectly with calculated Ge-E single bond lengths $(\mathrm{E}=\mathrm{S} 2.24 \AA$; Se 2.37 $\AA$; Te $2.57 \AA)^{[16]}$ and experimentally observed values of the corresponding chalcogenide-bridged dimers $\left\{\left[\left(\mathrm{Me}_{3} \mathrm{Si}\right)_{2} \mathrm{~N}\right]_{2} \mathrm{Ge}-\mu-\mathrm{E}\right\}_{2} \quad$ (av. Ge-E 2.2266(13) $\AA$ (S), $2.3678(4) \AA(\mathrm{Se}), 2.595(2) \AA(\mathrm{Te}))^{[17]}$ Moreover, the chalcogenide-bridged dimers $\left[\mathrm{Me}_{2} \mathrm{Si}(\mathrm{N} t-\mathrm{Bu})_{2} \mathrm{Ge}-\mu-\mathrm{E}\right]_{2}$, which were obtained from the reaction of the cyclic bis(amino)germylene $\left[\mathrm{Me}_{2} \mathrm{Si}(\mathrm{N} t-\mathrm{Bu})_{2} \mathrm{Ge}\right.$ with elemental chalcogens, show almost identical Ge-E bond lengths of $2.226(3) \AA(\mathrm{S}), 2.363(3) \AA(\mathrm{Se})$, and 2.592(5) $\AA$ (Te), respectively. ${ }^{[18]}$
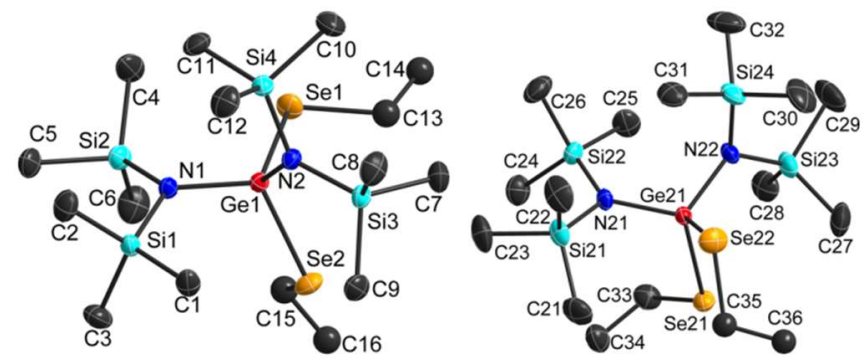

Figure 2. Solid state structure of 2. Non-H-atoms shown as therma ellipsoids at $50 \%$ probability levels, isotropically refined atoms shown as spheres, $\mathrm{H}$ atoms and second orientation of disordered parts omitted for clarity. Orientation of the molecules does not resemble their orientation in the asymmetric unit.

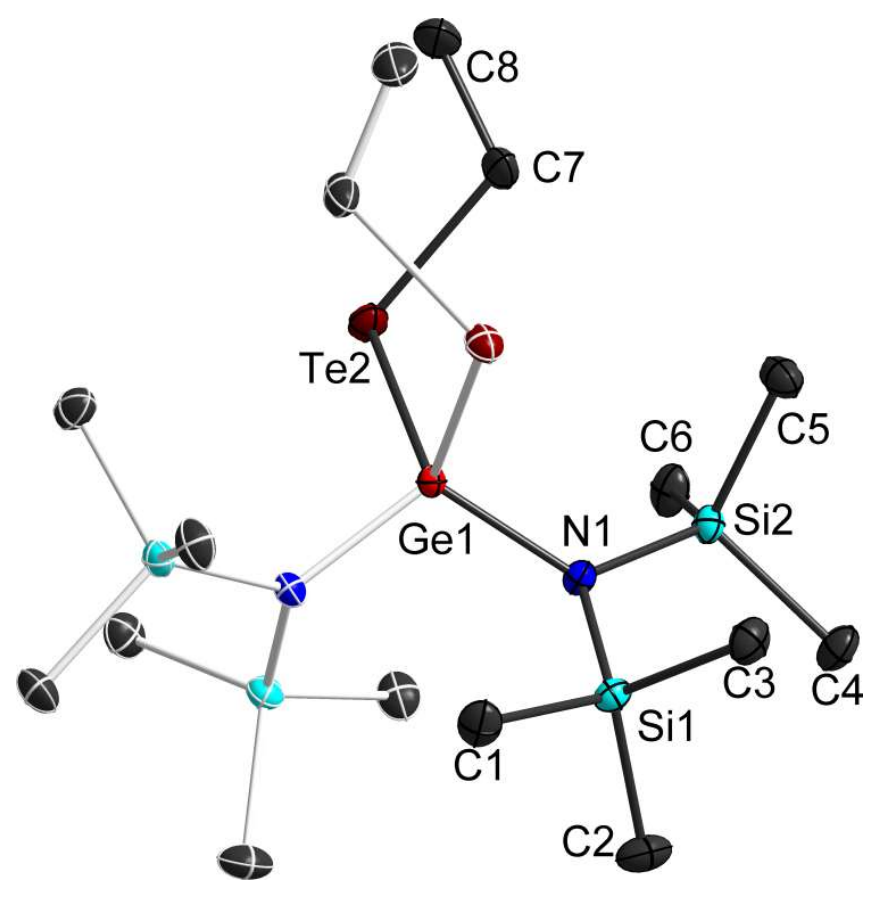

Figure 3. Solid state structure of 3. Non-H-atoms shown as thermal ellipsoids at $50 \%$ probability levels, $\mathrm{H}$ atoms omitted for clarity. Light colored part of the molecule generated by 2 -fold symmetry.

The average Ge-E bond lengths (av. 2.2335(5) $\AA$ 1; $2.3707(4) \AA \quad 2 ; 2.5803(2) \AA \quad 3)$ agree perfectly with calculated Ge-E single bond lengths $(\mathrm{E}=\mathrm{S} 2.24 \AA$; Se 2.37 $\AA$; Te $2.57 \AA)^{[16]}$ and experimentally observed values of the corresponding chalcogenide-bridged dimers $\left\{\left[\left(\mathrm{Me}_{3} \mathrm{Si}\right)_{2} \mathrm{~N}\right]_{2} \mathrm{Ge}-\mu-\mathrm{E}\right\}_{2} \quad$ (av. Ge-E 2.2266(13) $\AA \quad$ (S), $2.3678(4) \AA(\mathrm{Se}), 2.595(2) \AA(\mathrm{Te})) .^{[17]}$ Moreover, the chalcogenide-bridged dimers $\left[\mathrm{Me}_{2} \mathrm{Si}(\mathrm{N} t-\mathrm{Bu})_{2} \mathrm{Ge}-\mu-\mathrm{E}\right]_{2}$, which were obtained from the reaction of the cyclic bis(amino)germylene $\left[\mathrm{Me}_{2} \mathrm{Si}(\mathrm{N} t-\mathrm{Bu})_{2} \mathrm{Ge}\right.$ with elemental chalcogens, show almost identical Ge-E bond lengths of 2.226(3) $\AA(\mathrm{S}), 2.363(3) \AA(\mathrm{Se})$, and 2.592(5) $\AA$ (Te), respectively. ${ }^{[18]}$ In contrast, slightly elongated Ge-E bond 
lengths

were

reported

for $\left[\mathrm{C}_{6} \mathrm{H}_{11} \mathrm{NC}(\mathrm{Me}) \mathrm{NC}_{6} \mathrm{H}_{11}\right]_{2} \mathrm{Ge}\left[\mathrm{SeC}_{6} \mathrm{H}_{5}\right]_{2}(2.4744(17) \AA)^{[19]}$ and for a telluragermete containing sterically demanding substituents. ${ }^{[20]}$ Compared to the starting germylene $\left.\left[\left(\mathrm{Me}_{3} \mathrm{Si}\right)_{2} \mathrm{~N}\right)_{2}\right] \mathrm{Ge}(1.876(5) \AA),{ }^{[21]} \mathbf{1}-\mathbf{3}$ show slightly shorter Ge-N bond distances (av. value 1.8490(11) $\AA$ 1;

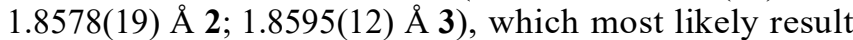
from the decreased radius of Ge(IV) compared to Ge(II) The N-Ge-N bond angle in $\mathbf{1}$ - $\mathbf{3}$ steadily decrease with increasing atomic number of the chalcogen atom $\left(113.30(5)^{\circ} \mathbf{1} ; 112.72(9)^{\circ} \mathbf{2} ; 109.62(8)^{\circ} \mathbf{3}\right)$, most likely resulting from the decreasing electronegativity of the chalcogen atoms, but are all widened compared to the germylene $\left.\left[\left(\mathrm{Me}_{3} \mathrm{Si}\right)_{2} \mathrm{~N}\right)_{2}\right] \mathrm{Ge}\left(107.1(2)^{\circ}\right)$. In contrast, the average E-Ge-E bond angles in $\mathbf{1}$ - $\mathbf{3}\left(105.06(2)^{\circ} \mathbf{1}\right.$; $\left.104.60(2)^{\circ} \mathbf{2} ; 105.87(2)^{\circ} \mathbf{3}\right)$ are almost identical.

Table 2. Selected bond lengths $[\AA]$ and angles $\left[{ }^{\circ}\right]$ of bis(ethylchalcogenato)germanes $\left.\left[\mathrm{Me}_{3} \mathrm{Si}\right)_{2} \mathrm{~N}\right]_{2} \mathrm{Ge}(\mathrm{EEt})_{2}(\mathrm{E}=\mathrm{S} \mathbf{1}, \mathrm{Se}$ 2, Te 3).

\begin{tabular}{|c|c|c|c|}
\hline & 1 & 2 & 3 \\
\hline \multirow[t]{4}{*}{ Ge-N } & $1.8457(11)$ & $1.8573(17)$ & $1.8595(12)$ \\
\hline & $1.8500(10)$ & $1.8582(18)$ & \\
\hline & $1.8500(11)$ & $1.8573(18)$ & \\
\hline & $1.8502(11)$ & $1.8585(19)$ & \\
\hline \multirow[t]{4}{*}{ Ge-E } & $2.2287(4)$ & $2.3667(3)$ & $2.5803(2)$ \\
\hline & $2.2359(5)$ & $2.3720(4)$ & \\
\hline & $2.2287(4)$ & $2.3613(3)$ & \\
\hline & $2.2408(4)$ & $2.3828(4)$ & \\
\hline \multirow[t]{2}{*}{$\mathrm{N}-\mathrm{Ge}-\mathrm{N}$} & $113.30(5)$ & $112.22(8)$ & $109.62(8)$ \\
\hline & $113.30(5)$ & $113.21(9)$ & \\
\hline \multirow[t]{8}{*}{ N-Ge-E } & $112.31(4)$ & $107.14(5)$ & $102.12(4)$ \\
\hline & $106.67(3)$ & $112.32(6)$ & $119.00(4)$ \\
\hline & $106.52(4)$ & $111.63(6)$ & \\
\hline & $111.90(4)$ & $107.29(5)$ & \\
\hline & $109.96(4)$ & $109.72(6)$ & \\
\hline & $112.20(4)$ & $113.10(6)$ & \\
\hline & $110.55(4)$ & $111.95(6)$ & \\
\hline & $106.22(4)$ & $105.28(6)$ & \\
\hline \multirow[t]{2}{*}{ E-Ge-E } & $105.967(14)$ & $106.125(12)$ & $105.866(11)$ \\
\hline & $104.142(15)$ & $103.072(13)$ & \\
\hline
\end{tabular}

[a] NMR spectra were recorded in $\mathrm{C}_{6} \mathrm{D}_{6}$.

We also expanded our systematic studies on reactions of the analogous bis[bis(trimethylsilyl]amido]stannylene, which is monomeric in gas phase and solid state. ${ }^{[2]}$ $\left.\left[\left(\mathrm{Me}_{3} \mathrm{Si}\right)_{2} \mathrm{~N}\right)_{2}\right] \mathrm{Sn}$ reacts with $\mathrm{Et}_{2} \mathrm{E}_{2}(\mathrm{E}=\mathrm{S}, \mathrm{Se}, \mathrm{Te})$ in hexane at ambient temperature with insertion of the stannylene into the E-E bond and subsequent formation of the expected stannanes $\left.\left[\left(\mathrm{Me}_{3} \mathrm{Si}\right)_{2} \mathrm{~N}\right)_{2}\right] \mathrm{Sn}(\mathrm{EEt})_{2}(\mathrm{E}=\mathrm{S}$ 4, Se 5, Te 6). Again, the progress of the reactions could be monitored by the color change of the solutions (yellow to colorless 4,5 ; red to yellow 6), which occurred within a minute. ${ }^{[15]}$ The ${ }^{1} \mathrm{H}$, ${ }^{13} \mathrm{C}$ as well as ${ }^{77} \mathrm{Se},{ }^{119} \mathrm{Sn}$ and ${ }^{127} \mathrm{Te}$ NMR spectra of $4-6$, which were also formed in almost quantitative yields, only showed a single resonance (table 1)
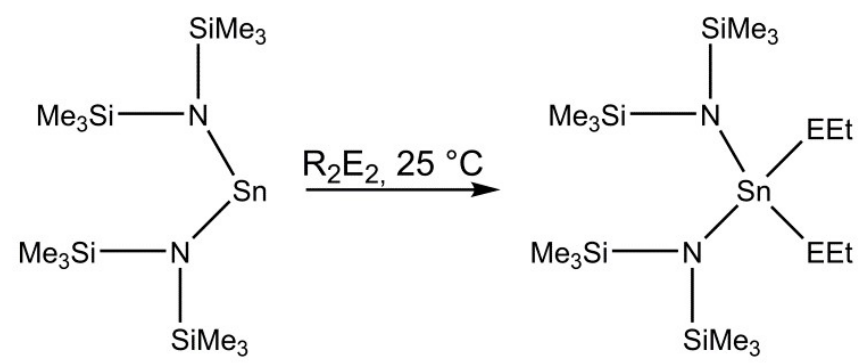

$E=S 4, \operatorname{Se} 5, \operatorname{Te} 6$

Scheme 2. Synthesis of $\mathbf{4}-\mathbf{6}$

Single crystals of $\mathbf{4}$ - $\mathbf{6}$ were obtained upon storage of freshly prepared solutions of $\mathbf{4}-\mathbf{6}$ in hexane at $-30{ }^{\circ} \mathrm{C}$ for 12h. $\mathbf{4}$ and $\mathbf{5}$ are isostructural to $\mathbf{1}$ and $\mathbf{2}$. $\mathbf{6}$ crystallizes in the monoclinc space group $P 2_{1} / c$.
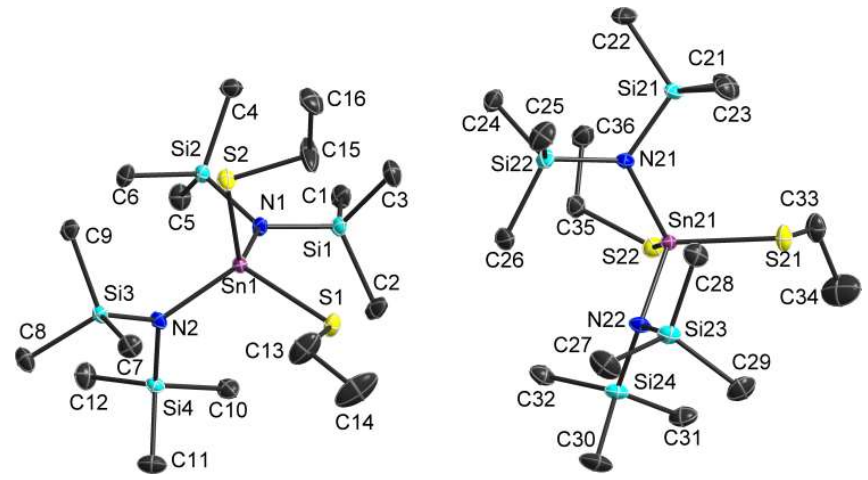

Figure 4. Solid state structure of 4 . Non-H-atoms shown as thermal ellipsoids at $50 \%$ probability levels for clarity.

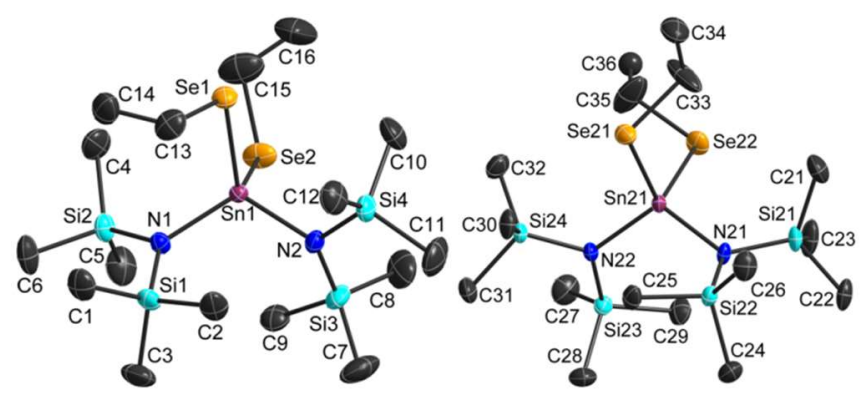

Figure 5. Solid state structure of $\mathbf{5}$, thermal ellipsoids are shown at $50 \%$ probability levels, $\mathrm{H}$ atom omitted for clarity. Orientation of the molecules does not resemble their orientation in the asymmetric unit.

The average Sn-E bond lengths increase with increasing atomic number of the chalcogen atom (av. 2.4032(4) $\AA$ 4; $2.5250(5) \AA 5$; 2.7480(5) $\AA$ 6). These values are slightly 
shorter than the calculated $\mathrm{Sn}-\mathrm{E}$ single bond lengths $(\mathrm{E}=\mathrm{S}$ $2.43 \AA$; Se $2.56 \AA$; Te $2.76 \AA),{ }^{[16]}$ but agree very well with the experimental values (CSD) for $\mathrm{Sn}-\mathrm{S}$ and $\mathrm{Sn}-\mathrm{Se}$ single bonds. ${ }^{[23]}$ Structurally characterized compounds containing terminal Sn-Te single bond such as 2,6-Mes $-\mathrm{C}_{6} \mathrm{H}_{3} \mathrm{TeSnMe}_{3}$ $(2.7484(4) \quad \AA),{ }^{[2]} \quad 2,6-\left(\mathrm{Me}_{2} \mathrm{~N}\right)_{2}-\mathrm{C}_{6} \mathrm{H}_{3} \mathrm{Sn}(\mathrm{TePh})_{2} \quad$ (av. $2.7425(5) \AA),{ }^{[10 a]}$ also show comparable Sn-Te bond lengths. In addition, the $\mathrm{Sn}-\mathrm{E}$ bond lengths in the chalcogenidebridged dimers $\left\{\left[\left(\mathrm{Me}_{3} \mathrm{Si}\right)_{2} \mathrm{~N}\right]_{2} \mathrm{Sn}-\mu-\mathrm{E}\right\}_{2}(\mathrm{E}=\mathrm{S} 2.416(6) \AA$; Se 2.541(1) $\AA$; Te 2.754(1) $\AA),{ }^{[25]}\left[\mathrm{Me}_{2} \mathrm{Si}(\mathrm{N} t-\mathrm{Bu})_{2} \mathrm{Sn}-\mu-\mathrm{E}\right]_{2}$ $(\mathrm{E}=\mathrm{Se} 2.536(3) \AA$; Te 2.741(3) $\AA),{ }^{[18]}$ and $t$-Bu $\left.\mathrm{Bu}_{2} \mathrm{Sn}-\mu-\mathrm{E}\right\}_{2}$ $(\mathrm{E}=\mathrm{S} 2.431(4), 2.422(4) \AA ;$ Se 2.551(2)) $\AA$; Te 2.756(1) $\AA)^{[26]}$ are nearly identical.

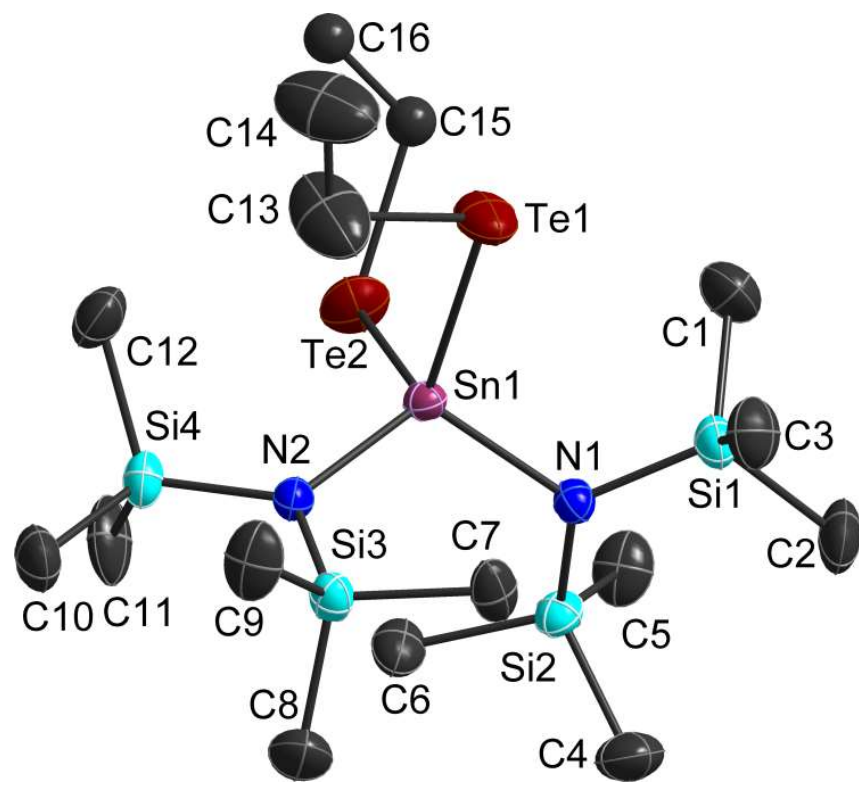

Figure 6. Solid state structure of 6, thermal ellipsoids are shown at $50 \%$ probability levels, isotropically refined atoms shown as spheres, $\mathrm{H}$ atoms and second orientation of disordered parts omitted for clarity.

The Sn-N bond distances (av. value 2.0422(10) $\AA$ 4; $2.045(2) \AA 5$; 2.063(4) $\AA$ 6) are shorter than those of the stannylene $\left.\left[\left(\mathrm{Me}_{3} \mathrm{Si}\right)_{2} \mathrm{~N}\right)_{2}\right] \mathrm{Sn}(2.092(6) \AA)^{[21]}$ as was expected due to the smaller radius of $\mathrm{Sn}(\mathrm{IV})$ compared to $\mathrm{Sn}$ (II). In contrast to the analogous germanes $\mathbf{1}-\mathbf{3}$, the N-Sn-N bond angle in $\mathbf{4}-\mathbf{6}$ are almost the same $\left(113.30(4)^{\circ} \mathbf{4}\right.$; $\left.112.60(10)^{\circ} \mathbf{5} ; 112.75(14)^{\circ} 6\right)$. Again, they are significantly widened compared to $\left.\left[\left(\mathrm{Me}_{3} \mathrm{Si}\right)_{2} \mathrm{~N}\right)_{2}\right] \mathrm{Sn}\left(104.7(2)^{\circ}\right)$. The average E-Sn-E bond angles slightly increase $\left(104.81(2)^{\circ} \mathbf{4}\right.$; $\left.104.82(2)^{\circ} \mathbf{5} ; 108.51(2)^{\circ} \mathbf{6}\right)$ with increasing atomic number of the chalcogen atom.

In sharp contrast to the reaction of the cyclic germylene $\mathrm{Me}_{2} \mathrm{Si}(\mathrm{N} t-\mathrm{Bu})_{2} \mathrm{Ge}^{[13]}$ both bis[bis(trimethylsilyl]amido]germylene $\left.\left(\mathrm{Me}_{3} \mathrm{Si}\right)_{2} \mathrm{~N}\right)_{2} \mathrm{Ge}$ and -stannylene $\left.\left(\mathrm{Me}_{3} \mathrm{Si}\right)_{2} \mathrm{~N}\right)_{2} \mathrm{Sn}$ only react with $\mathrm{E}_{2} \mathrm{Ph}_{2}$ with oxidative addition and formation of the corresponding germanes and stannanes $\left.\left(\mathrm{Me}_{3} \mathrm{Si}\right)_{2} \mathrm{~N}\right)_{2} \mathrm{M}(\mathrm{EPh})_{2}(\mathrm{M}=\mathrm{Ge}, \mathrm{Sn}, \mathrm{E}=\mathrm{S}, \mathrm{Se}, \mathrm{Te})$. This reaction behavior was expected since both compounds adopt monomeric structures in solution. As a consequence, the digermane formation as observed in the analogous reaction of $\mathrm{Me}_{2} \mathrm{Si}(\mathrm{N} t-\mathrm{Bu})_{2} \mathrm{Ge}$ most likely points to the formation of an at least weakly bonded dimer in solution, which consequently undergoes $2+2$ cycloaddition reactions.
Table 3. Selected bond lengths $[\AA]$ and angles $\left[{ }^{\circ}\right]$ of bis(ethylchalcogenato)stannanes $\left.\left[\mathrm{Me}_{3} \mathrm{Si}\right)_{2} \mathrm{~N}\right]_{2} \mathrm{Sn}(\mathrm{EEt})_{2}(\mathrm{E}=\mathrm{S} 4$, Se 5, Te 6).

\begin{tabular}{|c|c|c|c|}
\hline & 4 & 5 & 6 \\
\hline \multirow[t]{4}{*}{$\mathrm{Sn}-\mathrm{N}$} & $2.0399(10)$ & $2.045(2)$ & $2.069(3)$ \\
\hline & $2.0410(10)$ & $2.048(2)$ & $2.056(4)$ \\
\hline & $2.0432(10)$ & $2.043(2)$ & \\
\hline & $2.0448(10)$ & $2.044(2)$ & \\
\hline \multirow[t]{4}{*}{ Sn-E } & $2.4019(3)$ & $2.5182(4)$ & $2.7356(5)$ \\
\hline & $2.4070(4)$ & $2.5307(4)$ & $2.7643(5)$ \\
\hline & $2.3970(3)$ & $2.5226(4)$ & \\
\hline & $2.4070(3)$ & $2.5286(5)$ & \\
\hline \multirow[t]{2}{*}{ N-Sn-N } & $113.02(4)$ & $113.33(10)$ & $112.75(14)$ \\
\hline & $113.60(4)$ & $111.86(9)$ & \\
\hline \multirow[t]{8}{*}{ N-Sn-E } & $111.30(3)$ & $108.33(7)$ & $107.69(10)$ \\
\hline & $108.19(3)$ & $113.62(7)$ & $110.01(9)$ \\
\hline & $108.18(3)$ & $112.66(7)$ & $113.31(10)$ \\
\hline & $110.69(3)$ & $104.69(7)$ & $104.49(10)$ \\
\hline & $108.44(3)$ & $108.36(6)$ & \\
\hline & $112.96(3)$ & 111.61(7) & \\
\hline & $111.51(3)$ & $110.38(6)$ & \\
\hline & $105.52(3)$ & 108.67(6) & \\
\hline \multirow[t]{2}{*}{ E-Sn-E } & $105.187(12)$ & $103.848(14)$ & $108.507(17)$ \\
\hline & $104.439(12)$ & $105.790(13)$ & \\
\hline
\end{tabular}

\section{Conclusions}

Oxidative addition reactions of diethyldichalcogenanes $\mathrm{Et}_{2} \mathrm{E}_{2} \quad(\mathrm{E}=\mathrm{S}, \mathrm{Se}, \mathrm{Te})$ with bis[bis(trimethylsilyl)amido]germylene and -stannylene $\mathrm{M}\left[\mathrm{N}\left(\mathrm{SiMe}_{3}\right)_{2}\right]_{2}(\mathrm{M}=\mathrm{Ge}$, $\mathrm{Sn})$ yield the corresponding bis(diethylchalcogenato)germanes and stannanes $\mathbf{1}$ - 6. In contrast to analogous reactions of $\mathrm{Me}_{2} \mathrm{Si}(\mathrm{Nt}-\mathrm{Bu})_{2} \mathrm{Ge}$, no formation of digermanes was observed. 1 - $\mathbf{6}$ were characterized by heteronuclear NMR and IR spectroscopy and their solid state structures of 1 - $\mathbf{6}$ were determined by single crystal X-ray diffraction.

\section{Experimental Section}

All manipulations were performed under Ar. Solvents were carefully dried with $\mathrm{Na} / \mathrm{K}$ and degassed prior to use.

$\left[\left(\mathrm{Me}{ }_{3} \mathrm{Si}\right)_{2} \mathrm{~N}\right]_{2} \mathrm{Ge}$ and $\left[\left(\mathrm{Me}_{3} \mathrm{Si}\right)_{2} \mathrm{~N}\right]_{2} \mathrm{Sn}^{[14]}$ as well as $\mathrm{Et}_{2} \mathrm{Se}_{2}$ and $\mathrm{Et}_{2} \mathrm{Te}_{2}{ }^{[27]}$ were synthesized according literature procedures, whereas $\mathrm{Et}_{2} \mathrm{~S}_{2}$ was commercially available and distilled before use. ${ }^{1} \mathrm{H} N M R,{ }^{13} \mathrm{C}\left\{{ }^{1} \mathrm{H}\right\},{ }^{77} \mathrm{Se}\left\{{ }^{1} \mathrm{H}\right\},{ }^{119} \mathrm{Sn}\left\{{ }^{1} \mathrm{H}\right\}$, and ${ }^{127} \mathrm{Te}\left\{{ }^{1} \mathrm{H}\right\} \mathrm{NMR}$ spectra were recorded with a Bruker DMX 300 spectrometer and are referenced to internal $\mathrm{C}_{6} \mathrm{D}_{5} \mathrm{H}\left({ }^{1} \mathrm{H}\right.$ NMR: $\delta=7.16 \mathrm{ppm} ;{ }^{13} \mathrm{C}$ NMR: $\delta=128.0 \mathrm{ppm})$ and TMS $\left({ }^{77} \mathrm{Se}\left\{{ }^{1} \mathrm{H}\right\},{ }^{119} \mathrm{Sn}\left\{{ }^{1} \mathrm{H}\right\}\right.$, and $\left.{ }^{127} \mathrm{Te}\left\{{ }^{1} \mathrm{H}\right\}\right) .{ }^{[28]}$ IR spectra were recorded with an ALPHA-T FTIRspectrometer equipped with a single-reflection ATR sampling module. Melting points were measured in sealed capillaries. 
Elemental analyses were performed at the Elementaranalyse Labor of the University of Essen.

General procedure for the synthesis of 1 - 3 .

$0.5 \mathrm{mmol} \mathrm{Et}_{2} \mathrm{E}_{2}$ was added to a solution of $0.5 \mathrm{mmol}$

$\left.\left[(\mathrm{Me} 3 \mathrm{Si})_{2} \mathrm{~N}\right)\right]_{2} \mathrm{Ge}$ in $20 \mathrm{~mL}$ of hexane and stirred for $1 \mathrm{~h}$ at ambient

temperature. The solution was concentrated to $5 \mathrm{~mL}$ and stored at $30{ }^{\circ} \mathrm{C}$. Yellow crystals of $\mathbf{2 a}$ and colorless crystals of $\mathbf{2 b}$ and $\mathbf{2 c}$ were formed within $12 \mathrm{~h}$ and isolated by filtration.

$\left[\left(\mathrm{Me}_{3} \mathrm{Si}_{2}\right)_{2} \mathbf{N}_{2} \mathbf{G e}(\mathbf{S E t})_{2} \mathbf{1}\right.$ : Yield (isolated crystals): $0.25 \mathrm{~g}(96 \%)$, M.p. $200{ }^{\circ} \mathrm{C}$. Elemental analysis calcd (\%) for $\mathrm{C}_{16} \mathrm{H}_{46} \mathrm{GeN}_{2} \mathrm{Si}_{4} \mathrm{~S}_{2}$ (515.65): C 37.27, H 8.99, N 5.43; found C 37.20, H 8.81, N 5.11. ${ }^{1} \mathrm{H}$ NMR $\left(300 \mathrm{MHz}, \mathrm{C}_{6} \mathrm{D}_{6}, 298 \mathrm{~K}\right): \delta 0.47\left[\mathrm{~s}, 36 \mathrm{H}, \mathrm{Si}\left(\mathrm{CH}_{3}\right)_{3}\right], 1.20$ $\left[\mathrm{t},{ }^{3} \mathrm{~J}_{\mathrm{HH}}=7.5 \mathrm{~Hz}, 6 \mathrm{H}, \mathrm{CH}_{2}-\mathrm{CH}_{3}\right], 2.77\left[\mathrm{~s},{ }^{3} \mathrm{~J}_{\mathrm{HH}}=7.4 \mathrm{~Hz}, 4 \mathrm{H}, \mathrm{S}-\right.$ $\left.\mathrm{CH}_{2}\right] .{ }^{13} \mathrm{C}$ NMR $\left(75 \mathrm{MHz}, \mathrm{C}_{6} \mathrm{D}_{6}, 298 \mathrm{~K}\right): \delta 6.57$ [Si $\left.\left(\mathrm{CH}_{3}\right)_{3}\right], 17.18$ $\left[\mathrm{CH}_{2}-\mathrm{CH}_{3}\right], 24.74$ [S-CH $\mathrm{CH}_{2}$. IR $\left(\mathrm{v}, \mathrm{cm}^{-1}\right): 2961,1249,1093,1017$, $839,801,761,668,622,424 \mathrm{~cm}^{-1}$.

$\left[\left(\mathrm{Me}_{3} \mathbf{S i}\right)_{2} \mathbf{N}_{2} \mathbf{G e}(\mathbf{S e E t})_{2} 2\right.$ 2: Yield (isolated crystals): $0.28 \mathrm{~g}(93 \%)$, M.p. $197{ }^{\circ} \mathrm{C}$. Elemental analysis calcd (\%) for $\mathrm{C}_{16} \mathrm{H}_{46} \mathrm{GeN}_{2} \mathrm{Si}_{4} \mathrm{Se}_{2}$ (605.45): C 31.53, H 7.61, N 6.40; found C 32.10, H 7.66, N 4.43. ${ }^{1} \mathrm{H}$ NMR $\left(300 \mathrm{MHz}, \mathrm{C}_{6} \mathrm{D}_{6}, 298 \mathrm{~K}\right): \delta=0.49$ [s, 36H, $\left.\mathrm{Si}\left(\mathrm{CH}_{3}\right)_{3}\right]$, $1.29\left[\mathrm{t},{ }^{3} \mathrm{~J}_{\mathrm{HH}}=7.5 \mathrm{~Hz}, 6 \mathrm{H}, \mathrm{CH}_{2}-\mathrm{CH}_{3}\right], 2.77\left[\mathrm{~s},{ }^{3} \mathrm{~J}_{\mathrm{HH}}=7.5 \mathrm{~Hz}, 4 \mathrm{H}\right.$, $\left.\mathrm{Se}-\mathrm{CH}_{2}\right] .{ }^{13} \mathrm{C} \mathrm{NMR}\left(75 \mathrm{MHz}, \mathrm{C}_{6} \mathrm{D}_{6}, 298 \mathrm{~K}\right): \delta=6.70\left[\mathrm{Si}\left(\mathrm{CH}_{3}\right)_{3}\right]$, $16.98\left[\mathrm{CH}_{2}-\mathrm{CH}_{3}\right], 19.89\left[\mathrm{Se}-\mathrm{CH}_{2}\right] \cdot{ }^{77} \mathrm{Se} \mathrm{NMR}\left(57 \mathrm{MHz}, \mathrm{C}_{6} \mathrm{D}_{6}, 298\right.$ $\mathrm{K}): \delta=124.7 . \mathrm{IR}\left(\mathrm{v}, \mathrm{cm}^{-1}\right): 2959,1249,1224,1017,838,798,759$, $734,670,621,415 \mathrm{~cm}^{-1}$.

$\left[\left(\mathrm{Me}_{3} \mathrm{Si}_{2}\right)_{2} \mathbf{N}_{2} \mathbf{G e}(\mathrm{TeEt})_{2}\right.$ 3: Yield (isolated crystals): $0.32 \mathrm{~g} \mathrm{(90 \% ).}$ M.p. $153{ }^{\circ} \mathrm{C}$. Elemental analysis calcd (\%) for $\mathrm{C}_{16} \mathrm{H}_{46} \mathrm{GeN}_{2} \mathrm{Si}_{4} \mathrm{Te}{ }_{2}$ (706.73): C 27.19, H 6.56, N 3.96; found C 28.08, H 6.75, N 4.57. ${ }^{1} \mathrm{H}$ NMR $\left(300 \mathrm{MHz}, \mathrm{C}_{6} \mathrm{D}_{6}, 298 \mathrm{~K}\right): \delta=0.50\left[\mathrm{~s}, 36 \mathrm{H}, \mathrm{Si}\left(\mathrm{CH}_{3}\right)_{3}\right]$, $1.45\left[\mathrm{t},{ }^{3} \mathrm{~J}_{\mathrm{HH}}=7.7 \mathrm{~Hz}, 6 \mathrm{H}, \mathrm{CH}_{2}-\mathrm{CH}_{3}\right], 2.71\left[\mathrm{~s},{ }^{3} \mathrm{~J}_{\mathrm{HH}}=7.7 \mathrm{~Hz}, 4 \mathrm{H}\right.$, Te- $\left.\mathrm{CH}_{2}\right] .{ }^{13} \mathrm{C}$ NMR $\left(75 \mathrm{MHz}, \mathrm{C}_{6} \mathrm{D}_{6}, 298 \mathrm{~K}\right): \delta=4.05\left[\mathrm{Te}-\mathrm{CH}_{2}\right]$, $6.97\left[\mathrm{Si}\left(\mathrm{CH}_{3}\right)_{3}\right], 17.74\left[\mathrm{CH}_{2}-\mathrm{CH}_{3}\right] .{ }^{127} \mathrm{Te} \mathrm{NMR}\left(95 \mathrm{MHz}, \mathrm{C}_{6} \mathrm{D}_{6}\right.$, $298 \mathrm{~K}): \delta=162.3$. IR $\left(v, \mathrm{~cm}^{-1}\right): 2961,1246,1186,1028,794,721$, $687,667,549,506,414 \mathrm{~cm}^{-1}$.

General procedure for the synthesis of 4 - 6 .

$0.5 \mathrm{mmol} \mathrm{Et}_{2} \mathrm{E}_{2}$ was added to a solution of $0.5 \mathrm{mmol}$

$\left.\left[\left(\mathrm{Me}_{3} \mathrm{Si}\right)_{2} \mathrm{~N}\right)\right]_{2} \mathrm{Sn}$ in $20 \mathrm{~mL}$ of hexane and stirred for $1 \mathrm{~h}$ at ambient temperature. The solution was concentrated to $5 \mathrm{~mL}$ and stored at $30{ }^{\circ} \mathrm{C}$. Yellow crystals of $\mathbf{4}$ and colorless crystals of $\mathbf{5}$ and $\mathbf{6}$ were formed within $12 \mathrm{~h}$ and isolated by filtration.

$\left[\left(\mathrm{Me}_{3} \mathrm{Si}\right)_{2} \mathbf{N}_{2} \mathbf{S n}(\mathbf{S E t})_{2}\right.$ 4: Yield (isolated crystals): $0.26 \mathrm{~g}(95 \%)$, M.p. $182{ }^{\circ} \mathrm{C}$. Elemental analysis calcd (\%) for $\mathrm{C}_{16} \mathrm{H}_{46} \mathrm{SnN}_{2} \mathrm{Si}_{4} \mathrm{~S}_{2}$ (561.73): C 34.21, H 8.25, N 4.99; found C 69.08, H 7.70, N 10.67. ${ }^{1} \mathrm{H}$ NMR $\left(300 \mathrm{MHz}, \mathrm{C}_{6} \mathrm{D}_{6}, 298 \mathrm{~K}\right): \delta 0.42\left[\mathrm{~s}, 36 \mathrm{H}, \mathrm{Si}\left(\mathrm{CH}_{3}\right)_{3}\right], 1.23$ $\left[\mathrm{t},{ }^{3} \mathrm{~J}_{\mathrm{H}, \mathrm{H}}=7.6 \mathrm{~Hz},{ }^{4} \mathrm{~J}_{\mathrm{SnH}}=1.8 \mathrm{~Hz}, 6 \mathrm{H}, \mathrm{CH}_{2}-\mathrm{CH}_{3}\right], 2.83\left[\mathrm{q},{ }^{3} \mathrm{~J}_{\mathrm{HH}}=7.7\right.$ $\left.\mathrm{Hz},{ }^{3} \mathrm{~J}_{117 \mathrm{SnH}}=21.3 \mathrm{~Hz},{ }^{3} \mathrm{~J}_{119 \mathrm{SnH}}=22.4 \mathrm{~Hz}, 4 \mathrm{H}, \mathrm{Se}-\mathrm{CH}_{2}\right] .{ }^{13} \mathrm{C} \mathrm{NMR}$ $\left(75 \mathrm{MHz}, \mathrm{C}_{6} \mathrm{D}_{6}, 298 \mathrm{~K}\right): \delta 6.23\left[\mathrm{~s},{ }^{2} \mathrm{~J}_{\mathrm{SnC}}=27.9 \mathrm{~Hz}, \mathrm{Si}\left(\mathrm{CH}_{3}\right)_{3}\right]$, $\left.18.73\left[\mathrm{~s},{ }^{3} \mathrm{~J}_{\mathrm{SnC}}=20.3 \mathrm{~Hz}, \mathrm{CH}_{2}-\mathrm{CH}_{3}\right], 23.36\right)\left[\mathrm{s},{ }^{2} \mathrm{~J}_{\mathrm{SnC}}=10.4 \mathrm{~Hz}, \mathrm{~S}-\right.$ $\left.\mathrm{CH}_{2}\right] .{ }^{119} \mathrm{Sn}$ NMR $\left(110 \mathrm{MHz}, \mathrm{C}_{6} \mathrm{D}_{6}, 298 \mathrm{~K}\right): \delta=699.2$. IR $\left(v, \mathrm{~cm}^{-}\right.$ $\left.{ }^{1}\right): 2961,1249,1017,839,766,668,620,505,422 \mathrm{~cm}^{-1}$.

$\left[\left(\mathrm{Me}_{3} \mathrm{Si}\right)_{2} \mathrm{~N}_{2} \mathbf{S n}(\mathrm{SeEt})_{2}\right.$ 5: Yield (isolated crystals): $0.31 \mathrm{~g} \mathrm{(95 \% ),}$ M.p. $175^{\circ} \mathrm{C}$. Elemental analysis calcd (\%) for $\mathrm{C}_{16} \mathrm{H}_{46} \mathrm{SnN}_{2} \mathrm{Si}_{4} \mathrm{Se}_{2}$ (655.53): C 29.32, H 7.07, N 4.27; found C 29.51, H 7.73, N 4.20. ${ }^{1} \mathrm{H}$ NMR $\left(300 \mathrm{MHz}, \mathrm{C}_{6} \mathrm{D}_{6}, 298 \mathrm{~K}\right): \delta=0.48\left[\mathrm{~s}, 36 \mathrm{H}, \mathrm{Si}\left(\mathrm{CH}_{3}\right)_{3}\right]$, $1.31\left[\mathrm{t},{ }^{3} \mathrm{~J}_{\mathrm{HH}}=7.5 \mathrm{~Hz},{ }^{4} \mathrm{~J}_{\mathrm{SnH}}=1.9 \mathrm{~Hz}, 6 \mathrm{H}, \mathrm{CH}_{2}-\mathrm{CH}_{3}\right], 2.83\left[\mathrm{q},{ }^{3} \mathrm{~J}_{\mathrm{HH}}\right.$ $\left.=7.5 \mathrm{~Hz},{ }^{3} \mathrm{~J}_{117 \mathrm{SnH}}=22.5 \mathrm{~Hz},{ }^{3} \mathrm{~J}_{119 \mathrm{SnH}}=23.8 \mathrm{~Hz}, 4 \mathrm{H}, \mathrm{Se}-\mathrm{CH}_{2}\right] .{ }^{13} \mathrm{C}$ NMR $\left(75 \mathrm{MHz}, \mathrm{C}_{6} \mathrm{D}_{6}, 298 \mathrm{~K}\right): \delta=6.45\left[\mathrm{~s},{ }^{2} \mathrm{~J}_{\mathrm{SnC}}=27.9 \mathrm{~Hz}\right.$ $\left.\mathrm{Si}\left(\mathrm{CH}_{3}\right)_{3}\right], 17.25\left[\mathrm{~s},{ }^{3} \mathrm{~J}_{\mathrm{SnC}}=10.3 \mathrm{~Hz} \mathrm{CH}{ }_{2}-\mathrm{CH}_{3}\right], 18.35\left[\mathrm{~s},{ }^{2} \mathrm{~J}_{\mathrm{SnC}}=\right.$ $17.4 \mathrm{~Hz}, \mathrm{Se}-\mathrm{CH}_{2}$ ]. ${ }^{77} \mathrm{Se} \mathrm{NMR}\left(57 \mathrm{MHz}, \mathrm{C}_{6} \mathrm{D}_{6}, 298 \mathrm{~K}\right): \delta=39.7$. ${ }^{119} \mathrm{Sn}$ NMR $\left(110 \mathrm{MHz}, \mathrm{C}_{6} \mathrm{D}_{6}, 298 \mathrm{~K}\right): \delta=699.2 . \mathrm{IR}\left(v, \mathrm{~cm}^{-1}\right): 2960$, $1249,1017,798,723,670,508,420 \mathrm{~cm}^{-1}$.

$\left[\left(\mathrm{Me}_{3} \mathrm{Si}\right)_{2} \mathbf{N}\right]_{2} \mathbf{S n}(\mathrm{TeEt})_{2}$ 6: Yield (isolated crystals): $0.35 \mathrm{~g}(92 \%)$. M.p. $163{ }^{\circ} \mathrm{C}$. Elemental analysis calcd (\%) for $\mathrm{C}_{16} \mathrm{H}_{46} \mathrm{SnN}_{2} \mathrm{Si}_{4} \mathrm{Te}_{2}$
(752.81): C 34.21, H 8.25, N 4.99; found C 33.88, H 8.55, N 5.24. ${ }^{1} \mathrm{H}$ NMR $\left(300 \mathrm{MHz}, \mathrm{C}_{6} \mathrm{D}_{6}, 298 \mathrm{~K}\right): \delta=0.45\left[\mathrm{~s}, 36 \mathrm{H}, \mathrm{Si}\left(\mathrm{CH}_{3}\right)_{3}\right]$, $1.46\left[\mathrm{t},{ }^{3} \mathrm{~J}_{\mathrm{HH}}=7.4 \mathrm{~Hz},{ }^{4} \mathrm{~J}_{\mathrm{SnH}}=2.2 \mathrm{~Hz}, 6 \mathrm{H}, \mathrm{CH}_{2}-\mathrm{CH}_{3}\right], 2.76\left[\mathrm{q},{ }^{3} \mathrm{~J}_{\mathrm{HH}}\right.$ $\left.=7.4 \mathrm{~Hz},{ }^{3} \mathrm{~J}_{117 \mathrm{SnH}}=24.7 \mathrm{~Hz},{ }^{3} \mathrm{~J}_{119 \mathrm{SnH}}=26.0 \mathrm{~Hz}, 4 \mathrm{H}, \mathrm{Te}-\mathrm{CH}_{2}\right] .{ }^{13} \mathrm{C}$ NMR (75 MHz, $\left.\mathrm{C}_{6} \mathrm{D}_{6}, 298 \mathrm{~K}\right): \delta=-0.62\left[\mathrm{~s},{ }^{2} \mathrm{~J}_{\mathrm{SnC}}=8.5 \mathrm{~Hz}, \mathrm{Te}-\right.$ $\left.\mathrm{CH}_{2}\right], 6.83\left[\mathrm{~s},{ }^{2} \mathrm{~J}_{\mathrm{SnC}}=28.1 \mathrm{~Hz}, \mathrm{Si}\left(\mathrm{CH}_{3}\right)_{3}\right], 18.95\left[\mathrm{~s},{ }^{3} \mathrm{~J}_{\mathrm{SnC}}=13.1 \mathrm{~Hz}\right.$, $\left.\mathrm{CH}_{2}-\mathrm{CH}_{3}\right] .{ }^{119} \mathrm{Sn} \mathrm{NMR}\left(110 \mathrm{MHz}, \mathrm{C}_{6} \mathrm{D}_{6}, 298 \mathrm{~K}\right): \delta=699.2 .{ }^{127} \mathrm{Te}$ NMR (95 MHz, C6 6 6, $298 \mathrm{~K}): \delta=-15.5$. IR ( $\left(v, \mathrm{~cm}^{-1}\right)$ : 2961, 1246, $1150,780,721,687,553,515,414 \mathrm{~cm}^{-1}$.

Single crystal X-ray diffraction. Crystallographic data of 1 - 6, which were collected on a Bruker AXS SMART diffractometer (MoK $\alpha$ radiation, $\lambda=0.71073 \AA$ ) at $100(1) \mathrm{K}(1), 180(1) \mathrm{K}(2)$, 100(1) K (3), 100(1) K (4), 180(1) (5), and 200(1) K (6), are summarized in Table S1 (SI). The solid-state structures of of $\mathbf{1}$ - $\mathbf{6}$ are shown in Figures $1-6$, bond lengths and angles of of $\mathbf{1}-\mathbf{6}$ are summarized in tables 2 and 3. The structures were solved by Direct Methods (SHELXS-97) and refined anisotropically by full-matrix least-squares on $\mathrm{F}^{2}$ (SHELXL-97). ${ }^{[29,30]}$ Absorption corrections were performed semi-empirically from equivalent reflections on basis of multi-scans (Bruker AXS APEX2). Hydrogen atoms were refined using a riding model or rigid methyl groups. The $-\mathrm{E}-\mathrm{CH}_{2}-$ $\mathrm{CH}_{3}$ groups of 1, 2, 5 and $\mathbf{6}$ were disordered over two positions and not all of the carbon atoms could be refined anisotropically. The anisotropic displacement parameters of these groups in $\mathbf{4}$ also suggest disorder, however in this case it could not be resolved. The crystallographic data of $\mathbf{1}$ - $\mathbf{6}$ (excluding structure factors) have been deposited with the Cambridge Crystallographic Data Centre as supplementary publication nos. CCDC-1035552 (1), CCDC1035550 (2), CCDC-1035551 (3), CCDC-1035553 (4), CCDC1035555 (5) and CCDC-1035554 (6). Copies of the data can be obtained free of charge on application to CCDC, 12 Union Road, Cambridge, CB21EZ (fax: (+44) 1223/336033; e-mail: deposit@ccdc.cam-ak.uk).

Supporting Information (see footnote on the first page of this article): A CIF file giving X-ray crystallographic data of $\mathbf{1}$ - $\mathbf{6}$ as well as ${ }^{1} \mathrm{H},{ }^{13} \mathrm{C},{ }^{77} \mathrm{Se}{ }^{119} \mathrm{Sn}$ and ${ }^{127} \mathrm{Te}$ NMR spectra of $\mathbf{1}$ - 6 .

\section{Acknowledgement}

Stephan Schulz gratefully acknowledges financial support by the Mercator Research Center Ruhr (MERCUR).

[1] Y. Mizuhata, T. Sasamori, N. Tokitoh, Chem. Rev. 2009, 109, 3479 .

[2] a) C. Boehme, G. Frenking, J. Am. Chem. Soc. 1996, 118, 2039. b) M. Z. Kassaee, S. Soleimani-Amiri, M. Ghambarian, F. Boazar, E. Motamedi, Theochem. 2008, 849, 37. c) A. Bundhun, P. Ramasami, H. F. Schaefer III, J. Phys. Chem. A 2009, 113, 8080 .

[3] a) N. Tokitoh, R. Okazaki, Coord. Chem. Rev. 2000, 210, 251. b) O. Kühl, Coord. Chem. Rev. 2004, 248, 411. c) S. Nagendran, H. W. Roesky, Organometallics 2008, 27, 457. d) S. Yao, Y. Xiong, M. Driess Organometallics, 2011, 30, 1748. e) B. Blom, M. Stoelzel, M. Driess, Chem. Eur. J. 2013, 19, 40 .

[4] a) S. Schulz, S. Heimann, J. Friedrich, M. Engenhorst, G. Schierning, W. Assenmacher, Chem. Mater. 2012, 24, 2228; b) S. Schulz, S. Heimann, K. Kaiser, O. Prymak, W. Assenmacher, J. T. Brüggemann, B. Mallik, A.-V. Mudring, Inorg. Chem. 2013, 52, 14326; c) G. Bendt, S. Schulz, S Zastrow, K. Nielsch, Chem. Vap. Deposition 2013, 19, 235.

[5] M. Bouška, L. Střižík, L. Dostál, A. Růžička, A. Lyčka, L. Beneš, M. Vlček, Jan Přikryl, P. Knotek, T. Wágner, R. Jambor, Chem. Eur. J. 2013, 19, 1877. 
[6] a) Hao Zhang and Dmitri V. Talapin, Angew. Chem. Int. Ed. 2014, 53, 9126; b) L.-D. Zhao, S.-H. Lo, Y. Zhang, H. Sun, G. Tan, C. Uher, C. Wolverton, V. P. Dravid, M. G. Kanatzidis, Nature 2014, 508, 373.

[7] a) G. Pfister-Guillouzo, C. Guimon, Phosphorus and Sulphur 1985, 23, 197; b) P. B. Hitchcock, H. A. Jasim, M. F. Lappert, W.-P. Leung, A. K. Rai, R. E. Taylor, Polyhedron 1991, 10, 1203; c) D. Ellis, P. B. Hitchcock, M. F. Lappert, J. Chem Soc. Dalton Trans. 1992, 3397; d) I. Schranz, L. Grocholl, C. J. Carrow, L. Stahl, R. J. Staples, J. Organomet. Chem. 2008, 693, 1081; e) W.-P. Leung, W.-H. Kwok, L. T. C. Law, Z.-Y. Zhou, T. C. W. Mak, Chem. Commun. 1996, 505.

[8] a) M. Veith, S. Becker, V. Huch, Angew. Chem., Int. Ed. 1989, 28 (), pp. 1237; b) N. Tokitoh, T. Matsumoto, K. Manmaru, R. Okazaki, J. Am. Chem. Soc. 1993, 115, 8855; c) T. Matsumoto, N. Tokitoh, R. Okazaki, Angew. Chem. Int. Ed. Engl. 1994, 33, 2316; d) N. Tokitoh, T. Matsumoto, R. Okazaki, J. Am. Chem. Soc. 1997, 119, 2337; e) W.-P. Leung, W.-H. Kwok, Z.-Y. Zhou, T.C.W. Mak, Organometallics 2000, 19, 296; f) R. K. Siwatch, D. Yadav, G. Mukherjee, G. Rajaraman, S. Nagendran, Inorg. Chem. 2014, 53, 5073. For review articles see also: a) M.C. Kuchta, G. Parkin, Coord. Chem. Rev. 1998, 176, 323; b) R. Okazaki, N. Tokitoh, Acc. Chem. Res. 2000, 33, 625; R. C. Fischer, P. P. Power, Chem. Rev. 2010, 110, 3877.

[9] a) N. Tokitoh, T. Matsumoto, R. Okazaki, Tetrahedron Lett. 1992, 33, 2531. b) T. Matsumoto, N. Tokitoh, R. Okazaki, Organometallics 1995, 14, 1008.

[10] a) P. B. Hitchcock, M. F. Lappert, L. J.-M. Pierssens, A. V. Protchenko, P. G. H. Uiterweerd, J. Chem. Soc. Dalton Trans. 2009, 4578; b) M. Bouška, L. Dostal, A. Růžička, L. Beneš, R. Jambor, Chem. Eur. J. 2011, 17, 450; c) M. Bouška, L. Dostal, F. de Proft, A. Růžička, A. Lyčka, R. Jambor, Chem Eur. J. 2011, 17, 455; d) M. Bouška, L. Dostál, Z. Padělková, A. Lyčka, S. Herres-Pawlis, K. Jurkschat, R. Jambor, Angew. Chem. Int. Ed. 2012, 51, 3478; e) E. Moos, T. Augenstein, D. Garnier, F. Breher, Can. J. Chem. 2014, 92, 574

[11] S. R. Foley, G. P. A. Yap and D. S. Richeson, J. Chem. Soc., Dalton Trans. 2000, 1663.

[12] a) M. Wagner, C. Dietz, M. Bouška, L. Dostál, Z. Padělková, R. Jambor and K. Jurkschat, Organometallics, 2013, 32, 4973; b) P. Šimon, R. Jambor, A. Růžička, L. Dostál, Organometallics 2013, 32, 239; c) P. Śimon, R. Jambor, A. Růžička, L. Dostál, J. Organomet. Chem. 2013, 740, 98; d) M. Bouška, M. Novák, L. Dostál, A. Rủžička, T. Mikysek, R. Metelka, R. Jambor, Eur. J. Inorg. Chem. 2014, 310; e) S. Gondzik, S. Schulz, D. Bläser, C. Wölper, J. Chem. Soc. Chem. Commun. 2014, 50, 1189 .

[13] P. Steiniger, G. Bendt, D. Bläser, C. Wölper, S. Schulz, J. Chem. Soc. Chem. Commun. 2014, 50, 15461.

[14] M. J. S. Gynane, D. H. Harris, M. F. Lappert, P. P. Power, P. Rivière, M. Rivière-Baudet, J. Chem. Soc., Dalton Trans. 1977, 2004.

[15] The colors of the starting reagents are as follows: $\left.\left[\left(\mathrm{Me} \mathrm{Si}_{2}\right)_{2} \mathrm{~N}\right)\right]_{2} \mathrm{Ge}$ light yellow; $\left.\left[\left(\mathrm{Me}_{3} \mathrm{Si}\right)_{2} \mathrm{~N}\right)\right]_{2} \mathrm{Sn}$ orange; $\mathrm{Et}_{2} \mathrm{~S}_{2}$ colorless, $\mathrm{Et}_{2} \mathrm{Se}_{2}$ orange, $\mathrm{Et}_{2} \mathrm{Te}_{2}$ red.

[16] P. Pyykkö, M. Atsumi, Chem.-Eur. J., 2009, 15, 186.

[17] a) G. L. Wegner, A. Jockisch, A. Schier, H. Schmidbaur, $Z$. Naturforsch 2000, 55B, 347; b) P. B. Hitchcock, H. A. Jasim, M. F. Lappert, Wing-Por Leung, A. K. Rai, R. E. Taylor, Polyhedron 1991, 10, 1203.

[18] M. Veith, M. Nötzel, L. Stahl, V. Huch, Z. Anorg. Allg. Chem. 1994, 620, 1264.

[19] S. R. Foley, G. P. A. Yap, D. S. Richeson, J. Chem. Soc., Dalton Trans., 2000, 1663.

[20] T. Sasamori, T. Sasaki, N. Takeda, N. Tokitoh, Organometallics 2005, 24, 612.

[21] R. W. Chorley, P. B. Hitchcock, M. F. Lappert, Wing-Por Leung, P. P. Power, M. M. Olmstead, Inorg. Chim. Acta 1992, 198, 203.
[22] T. Fjeldberg, H. Hope, M. F. Lappert, P. P. Power, A. J. Thorne, J. Chem. Soc. Dalton Trans. 1986, 1551.

[23] CSD search for fragment Sn-S-C, $\operatorname{cn}(\mathrm{Sn})=4, \mathrm{Cn}(\mathrm{S})=2,726$ bonds from 336 hits, 2.357 to $2.819 \AA$, mean 2.462(2) $\AA$ median at $2.457 \AA$. CSD search for fragment Sn-Se-C, cn $(\mathrm{Sn})$ $=4, \mathrm{Cn}(\mathrm{Se})=2,41$ bonds from 20 hits, 2.478 to $2.746 \AA$, mean 2.552(9) $\AA$, median at $2.539 \AA$.

[24] H. Poleschner, S. Ellrodt, M. Malischewski, J. Nakatsuji, C. Rohner, K. Seppelt, Angew. Chem. Int. Ed. 2012, 51, 419.

[25] P. B. Hitchcock, E. Jang, M. F. Lappert, J. Chem. Soc., Dalton Trans., 1995, 3179.

[26] H. Puff, B. Breuer, W. Schuh, R. Sievers, R. Zimmer, J. Organomet. Chem. 1987, 332, 279.

[27] Ming T. Chen, John W. George, J. Organometal. Chem. 1968, 12, 401-403.

[28] R. K. Harris, E. D. Becker, S. M. Cabral de Menezes, R. Goodfellow, P. Granger, Magn. Reson. Chem. 2002, 40, 489.

[29] G. M. Sheldrick, Acta Crystallogr. Sect. A 1990, 46, 467.

[30] G. M. Sheldrick, SHELXL-97, Program for the Refinement of Crystal Structures University of Göttingen, Göttingen (Germany) 1997 (see also: G. M. Sheldrick, Acta Crystallogr. Sect. A 2008, 64, 112.).

Received: ((will be filled in by the editorial staff)) Published online: ((will be filled in by the editorial staff)) 


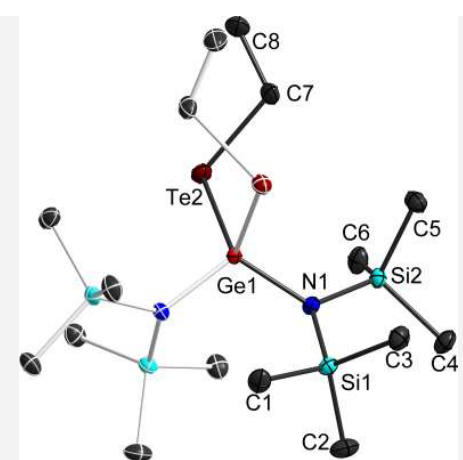

Georg Bendt, Silvia Lapsien, Phillip Steiniger, Dieter Bläser, Christoph Wölper and Stephan Schulz* ....... Page No. - Page No.

Oxidative Addition of Diethylchalcogenanes to Lapperts Germylene and Stannylene 
DuEPublico

Duisburg-Essen Publications online

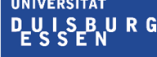

offen im Denken

$\mathbf{U b} \mid \begin{aligned} & \text { universitäts } \\ & \text { bibliothek }\end{aligned}$

This text is made available via DuEPublico, the institutional repository of the University of Duisburg-Essen. This version may eventually differ from another version distributed by a commercial publisher.

DOI: $\quad 10.1002 /$ zaac.201500023

URN: urn:nbn:de:hbz:464-20201125-104328-9

This is the peer reviewed version of the following article: Z. Anorg. Allg . Chem. 2015, 641, 797-902, which has been published in final form at:

https://doi.org/10.1002/zaac.201500023

All rights reserved. 